\title{
Design, Simulation, Modeling, and Implementation of a Square Helmholtz Coil in Contrast with a Circular Coil for MRI Applications
}

\author{
Sidi M. Ahmed Ghaly \\ Electrical Engineering Department, College of Engineering \\ Al Imam Mohammad Ibn Saud Islamic University \\ Riyadh, Saudi Arabia \\ smghaly@imamu.edu.sa
}

\author{
Mohammad Obaidullah Khan \\ Electrical Engineering Department, College of Engineering \\ Al Imam Mohammad Ibn Saud Islamic University \\ Riyadh, Saudi Arabia \\ dearkhan@gmail.com
}

\begin{abstract}
This paper focuses on Helmholtz-type coils that can produce a second-order homogeneity field to be used for Magnetic Resonance Imaging (MRI) applications. A Helmholtz coil is a device used for producing a region of a nearly uniform magnetic field. It consists of two identical magnetic coils that are placed symmetrically along a common axis, one on either side of the experimental area, separated by a distance equal to the radius of the circular coil and half-length of the side of the square coils. Each coil carries an equal electrical current flowing in the same direction. The main objective of this article is to calculate the magnetic field provided by the coils at any point in space and to show and compare the uniform magnetic field induced by the square and circular Helmholtz coils. With the aid of MATLAB simulation tool, mathematical equations are simulated to demonstrate the axial magnetic field produced by one and two loops. Also, the design and simulation of electrical modeling for square and circular Helmholtz coils are performed using PSPICE. Finally, these coils are realized and tested experimentally, and the results for square and circular Helmholtz coils are compared.
\end{abstract}

Keywords-square and circular Helmholtz coil; radiofrequency coils; modeling; simulation; MATLAB; PSPICE; electromagnetic field measurement; impedance measurements; MRI

\section{INTRODUCTION}

A Helmholtz coil is a device used for producing a region of nearly uniform magnetic field [1-2]. It consists of two identical circular magnetic coils that are placed symmetrically, one on either side of the experimental area along a common axis, and separated by a distance $d$ equal to the radius $R$ of the coil. The currents in the coils are equal and flowing in the same direction. Several variations exist in the coil topology, including the use of rectangular coils, and different number of coils. However, a two-coil Helmholtz pair is the standard model, with coils that are circular in shape and flat on the sides. In such a device, an electric current is passed through the coils to create a very uniform magnetic field. Helmholtz coils are used for a variety of purposes. They were used in an argon tube experiment to measure the charge to mass ratio of electrons [3]. They are often used to measure the strength and fields of permanent magnets [4-5]. To do this, the coil pair is connected to a flux meter, a device which contains measuring coils and electronics that evaluate the change of voltage in the measuring coils to calculate the overall magnetic flux. In some applications, a Helmholtz coil is used to cancel out Earth's magnetic field, producing a region with magnetic field intensity close to zero. This can be used to see how electrical charges and magnetic fields operate when not affected by the gravitational pull of the earth or other celestial bodies [6-7].

This work is focused on radiofrequency Helmholtz type coils that can be used for MRI applications. In particular, the design, modeling, and comparison of circular and square Helmholtz coils for magnetic resonance imaging render certain simplicity of implementation besides generating good homogeneity of the radiofrequency electromagnetic field.

\section{MAGNETIC FIELD CALCULATION AND ELECTRICAL MODELING}

\section{A. Circular Helmholtz Coil}

Consider a circular Helmholtz coil of radius $R$, separated by a distance $l$ as illustrated in Figure 1. There is a steady current flow $I$ in the same direction through each coil. Let the magnetic field $B_{1}$ be calculated on the axis at a distance $z$ from the center of one coil.

(a)

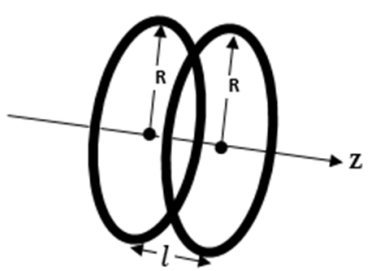

(b)

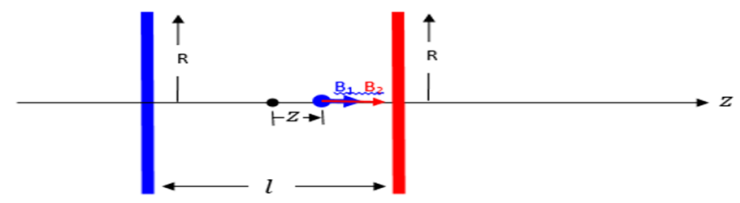

Fig. 1. Helmholtz circular coil arrangement: (a) 3D, (b) 2D 


$$
\begin{gathered}
\frac{\mu_{0} N I R^{2}}{2}\left[\frac{1}{\left[\left(z+\frac{l}{2}\right)^{2}+R^{2}\right]^{3 / 2}}+\frac{1}{\left[\left(z-\frac{l}{2}\right)^{2}+R^{2}\right]^{3 / 2}}\right]
\end{gathered}
$$

The magnetic field strength at $z=0$ and $l=R$ is given by:

$$
B_{Z}(0)=\frac{\mu_{0} N I R^{2}}{(5 / 4)^{3 / 2} R}
$$

\section{B. Double Square Coil (Square Helmholtz Coil)}

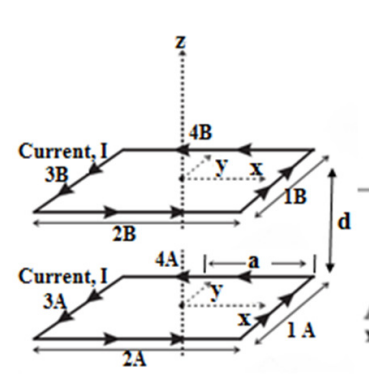

(a)

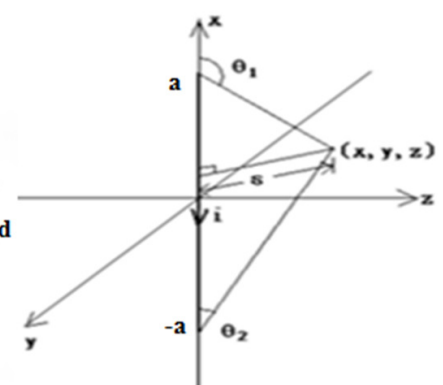

(b)
Fig. 2. (a) Square Helmholtz coil, (b) A current-carrying vertical eire

Consider two square coils arranged as a Helmholtz coil, as illustrated in Figure 2(a). The magnetic field produced by the side $3 \mathrm{~A}$ can be obtained by the same rotation on that by the side $2 \mathrm{~A}$. Similarly, the rotation on the field by the side $3 \mathrm{~A}$ gives the field by the side 4A. The field by the other coil (coil B) can then be obtained by simply replacing every $d$ in the equation by $-d$. The total field can be obtained by superposition of all the contributions of the wire segments. The separation between the two square coils is critical in the construction of the Helmholtz coil. Consider the field along the z-axis, which points along the $\mathrm{z}$-direction only [8-10]. By Taylor expansion around $z=0$, we have:

$$
B(z)=B(0)+\frac{d B}{d z}(0) z+\frac{1}{2} \frac{d^{2} B}{d z^{2}}(0) z^{2}+O\left(z^{3}\right)
$$

For small $z(0<z<1)$, the higher the order of the term is, the smaller the contribution this term has to the field at that point. By symmetry, $B(z)$ is symmetric about the $z=0$. Therefore, $B(z)$ is an even function, and $\frac{d B}{d z}(0)=0$. The term that follows immediately after $B(0)$ should be the second order term. In order to have a uniform field around the center of the Helmholtz coil, the contribution by this second order is suppressed to zero. This means that the condition for uniformity is:

$$
\frac{d^{2} B}{d z^{2}}(0)=0
$$

Along the $z$-axis, the magnetic field is given by:

$$
\begin{gathered}
B(z)=\frac{2 \mu_{0} I a^{2}}{\pi}\left[\frac{1}{\left[a^{2}+(z-d / 2)^{2}\right] \sqrt{2 a^{2}+(z-d / 2)^{2}}}+\right. \\
\left.\frac{1}{\left[a^{2}+(z-d / 2)^{2}\right] \sqrt{2 a^{2}+(z-d / 2)^{2}}}\right]
\end{gathered}
$$

The mutual inductance for the circular and square Helmholtz's loops are calculated based on the electrical modeling illustrated below:

$$
M=\frac{\mu_{0} \mu_{r} N_{1} N_{2} A}{l}
$$

where $\mu_{o}$ is the permeability of free space $\left(4 \pi \times 10^{-7}\right), \mu_{r}$ is the relative permeability of the soft iron core, $N$ is in the number of coil turns, $A$ is in the cross-sectional area in $\mathrm{m}^{2}$, and $l$ is the length of the coil in meters.

The design values for the typical parameters used in the square and circular Helmholtz coil setups are shown in Table I.

TABLE I. TYPICAL DESIGN VALUES FOR CIRCULAR AND SQUARE HELMHOLTZ COILS

\begin{tabular}{|c|c|c|}
\hline Parameter & Circular & Square \\
\hline $\begin{array}{c}M=\frac{\mu_{0} \mu_{r} N_{1} N_{2} A}{l} \\
\text { Mutual Inductance }\end{array}$ & $8.004 n \mathrm{H}$ & $6.283 n \mathrm{H}$ \\
\hline $\begin{array}{c}\text { Coupling Coefficient for } L 1=L 2: \\
M=K \sqrt{L_{1} \cdot L_{2}}\end{array}$ & 0.023 & 0.016 \\
\hline Resonant frequency $f_{0}$ & $f_{0}=99 \mathrm{MHz}$ & $f_{0}=102 \mathrm{MHz}$ \\
\hline Self frequency & $107 \mathrm{MHz}$ & $107 \mathrm{MHz}$ \\
\hline Resistance: $R=\frac{\rho l}{A}$ & $2.64 \mu \Omega$ & $3.36 \mu \Omega$ \\
\hline Inductance: & $0.3529 \mu \mathrm{H}$ & $0.3863 \mu \mathrm{H}$ \\
\hline$\mu_{0} a_{1} \ln \left(\frac{8 a_{1}}{R}-1.75\right)$ & $7.323 \mathrm{pF}$ & $6.899 \mathrm{pF}$ \\
\hline Capacitance: $C=\frac{1}{(2 \pi f)^{2} L}$ & &
\end{tabular}

\section{FIELD CALCULATION AND PATTERN PLOT}

To calculate the electromagnetic field $\left(B_{1}\right)$ produced by the proposed square and circular Helmholtz structures which consist of two symmetrical elements and have small dimensions compared to the wavelength, the superposition principle can be applied to obtain the total field as indicated in (2) and (5) for circular and square patterns [11-13].

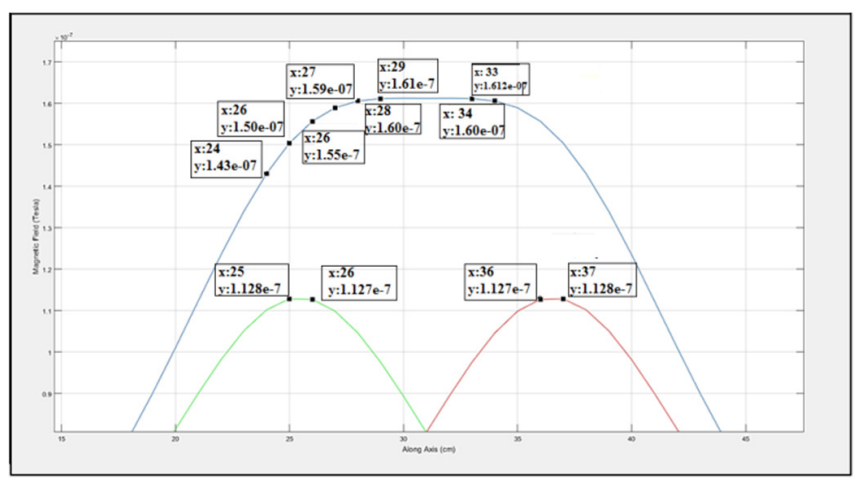

Fig. 3. Uniform magnetic field pattern between square coils

The simulation code for the double square coil is programmed in MATLAB for a typical input data of $I=1 \mathrm{~A}$, $d=2.77 \mathrm{~cm}, \quad a=5 \mathrm{~cm}, \quad N=1$, and $\mu_{0}=4 \pi \times 10^{-7} \mathrm{mkgs}^{-2} \mathrm{~A}^{-2}$. The simulation result is shown in Figure 3, where each coil has a magnetic field pattern indicated by red and green color patterns 
and the summation of these patterns is shown with blue color. It was noted that the best distance between the two coils separation was equal to $2.7 \mathrm{~cm}$, giving the maximum uniform magnetic field pattern corresponding to this input data. And it was also noted that a few (5) points that appear on constant magnetic field region as depicted in Figure 3 were obtained. Similarly, the simulation code for the double circular coil is developed in MATLAB for a typical input data of $I=1 \mathrm{~A}$, $d=2.56 \mathrm{~cm}, \quad R=5 \mathrm{~cm}, \quad N=1$, and $\mu_{0}=4 \pi \times 10^{-7} \mathrm{mkgs}^{-2} \mathrm{~A}^{-2}$. The simulation result is shown in Figure 4 where each coil has a magnetic field pattern indicated by red and green color and the summation of these patterns is shown with blue color. It was noted that the best distance between the two coils separation was equal $2.56 \mathrm{~cm}$, for which it gives the maximum uniform magnetic field pattern corresponding to this input data. And it was also noted that there are 3 points obtained that appear on constant magnetic field pattern. Figure 5 illustrates the normalized axial profiles of the $B_{1}$ field for the proposed square and circular structures where $Y$ is along the coil axis in the same reference. The magnetic field is calculated by the Biot-Savart law and normalized to its maximum value.

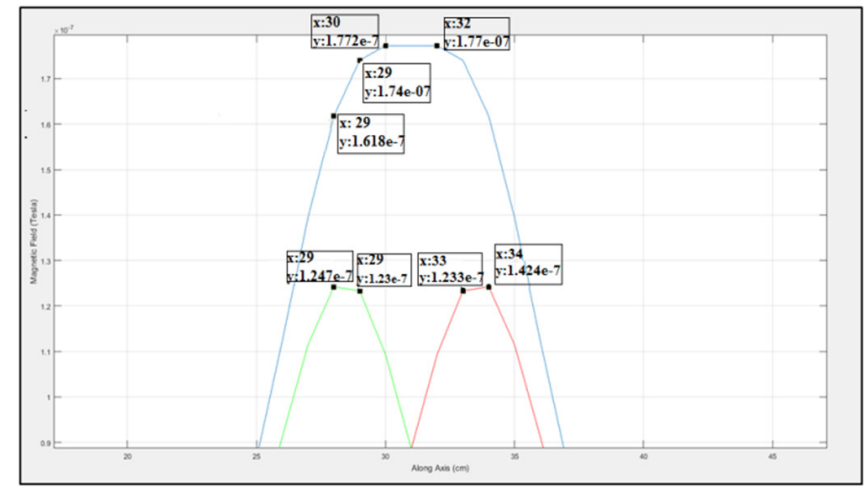

Fig. 4. Uniform magnetic field pattern between circular coils

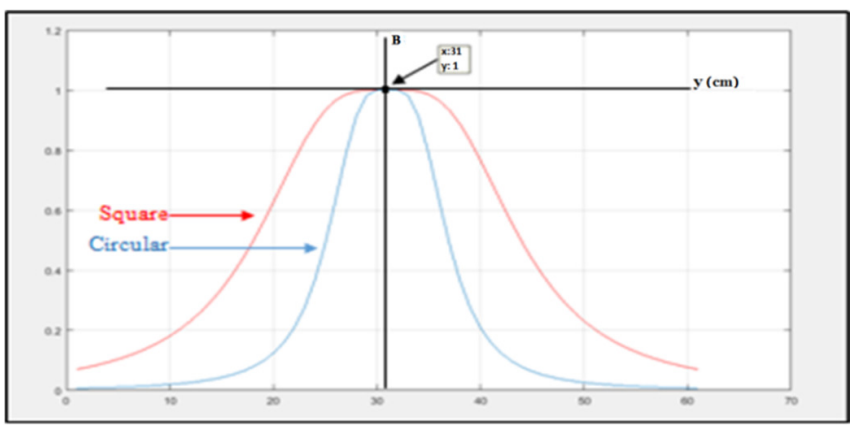

Fig. 5. Normalized magnetic field pattern for square and circular coils

\section{SimUlations}

In this part, the electrical response of the system is investigated for the proposed structures (circular and square). The system consists of two coaxial wire loops whose coupling mode is inductive coupling between all loops and capacitive coupling between adjacent left or right loops. Each one consists of identical tuned circuits resonating at the same frequency.
The proposed coil is matched using an inductive coupling [14]. This kind of matching uses an attack loop with the same dimensions with the first loop of the proposed coil, and this attack loop is tuned to $f_{l}$, as shown in Figure 6. The attack coil is inductively coupled with the two loops of the structure. The matching of the coil at $50 \Omega$ is performed by optimizing the coupling loop position. The simulation of the matching at $50 \Omega$ gives the corresponding distance between the attack loop and the circulator of the square Helmholtz coils [16-18]. The typical distances measured for circular and square coils are equal to $2.56 \mathrm{~cm}$ and $2.77 \mathrm{~cm}$ respectively. Figure 7 illustrates the simulation result of PSPICE for Matching to $50 \Omega$ for the circular single and double mode coil at $f_{0}=99 \mathrm{MHz}$. The inductive coupling results in an impedance inversion, the series resonance modes become parallel, and the simulation allows the user to check the design values for the circuit elements in the structure thoroughly. Similarly, for square Helmholtz coil PSPICE simulation is carried out, and the results are noted graphically.

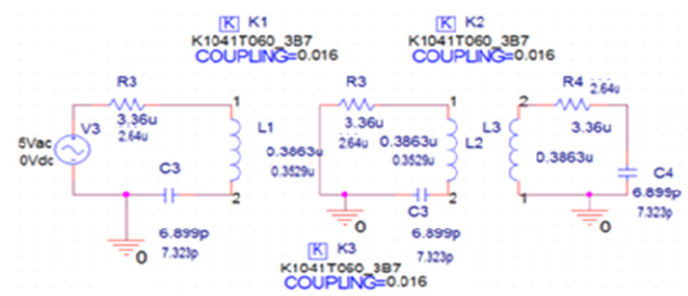

Fig. 6. SPICE model for matching of the square and circular Helmholtz coils with inductive coupling

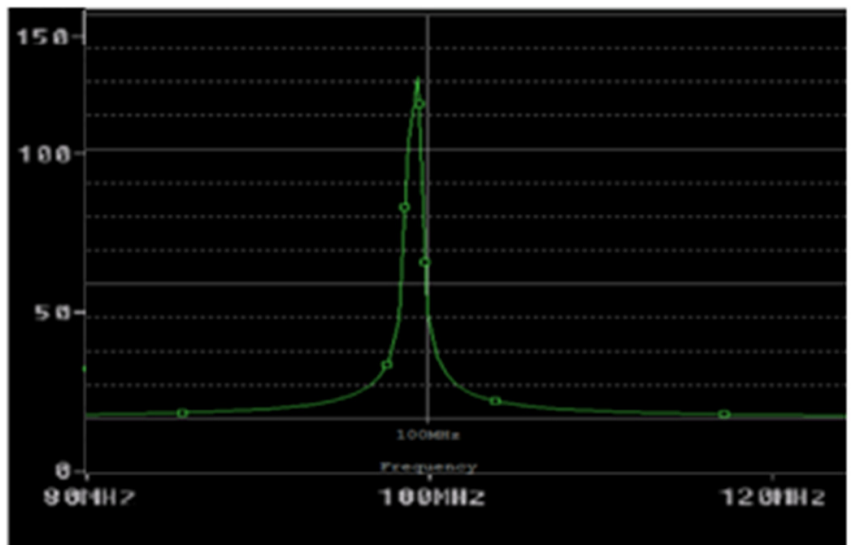

Fig. 7. Simulation of the matching to $50 \Omega$ for the circular and square single mode coils at resonant frequencies: $\mathrm{X}$-axis represents the frequencies, and $\mathrm{Y}$-axis represents the magnitude of the input impedance

\section{EXPERIMENTAL RESULTS}

In this section, the experimental results obtained with the implemented circular and square Helmholtz coils are demonstrated. The specimen tests conducted in this experiment are out-of-field tests (in the air) using a network analyzer. These tests were facilitated to carry out relative measurements of the radiofrequency electromagnetic field $\left(B_{1}\right)$ along the main axis of the coils and to compare them with the theoretical results [15]. 


\section{A. Prototype Dimensions}

To facilitate comparisons, both the circular and the square Helmholtz coils have the typical precise dimensions reported in Table II. Photographs of the developed coils can be seen in Figure 8 .

TABLE II. PRECISE DIMENSIONS OF CIRCULAR AND SQUARE HELMHOLTZ COILS

\begin{tabular}{|c|c|c|}
\hline Coil structure & Parameters & Distance between loops \\
\hline Circular & $R=5 \mathrm{~cm}$ & $2.56 \mathrm{~cm}$ \\
\hline Square & $l=5 \mathrm{~cm}, b=5 \mathrm{~cm}$ & $2.77 \mathrm{~cm}$ \\
\hline
\end{tabular}

(a)

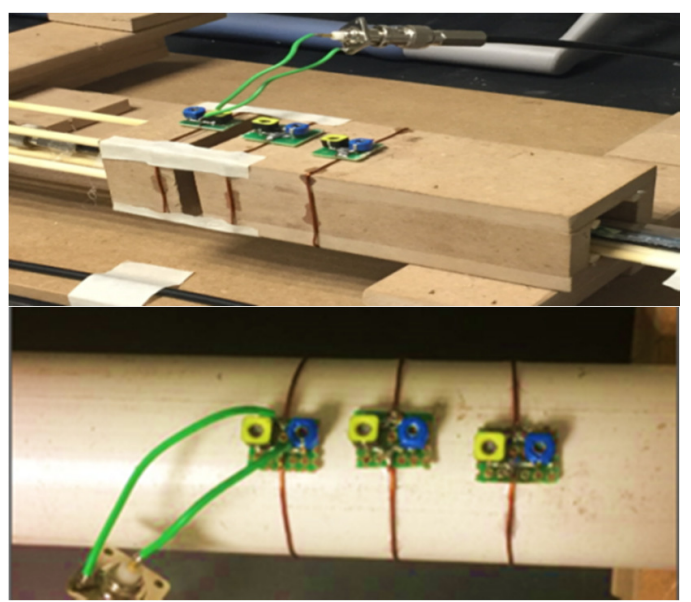

Fig. 8. (a) Square, (b) Helmholtz coils

\section{B. Matching and Tuning of the Coils}

It is possible to proceed directly to the tuning of the coils, but this requires a certain control of the various influential parameters (stepwise adjustment of the tuning capacitors). The tuning of each loop at $f_{0}$ is performed individually by shorting the other loops and bringing it closer to a test loop. By predetermination of $f_{0}$, a vacuum presetting of the coil is carried out. Figure 9 shows a photograph of the tuning of the circular coil obtained with impedance measurements using a Network Analyzer. The variations of the module and phase of the input impedance are seen by the source as a function of the frequency. The same is repeated for the square coil.

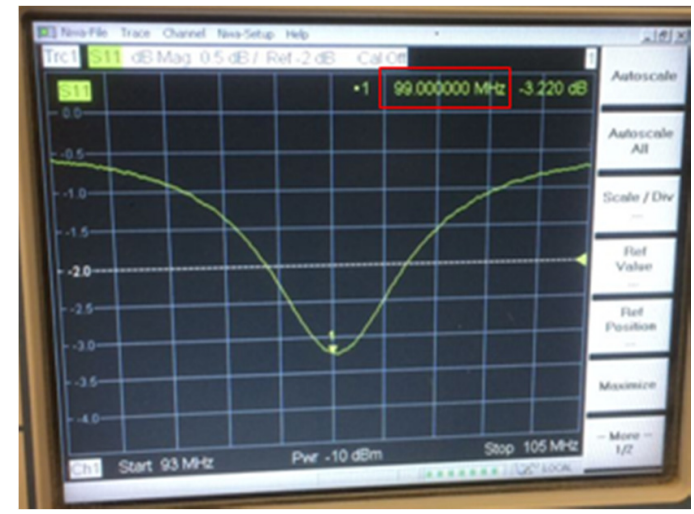

Fig. 9. Typical screen shot illustrating the resonance frequency for the circular Helmholtz coil in the Network Analyzer
Two parallel resonance frequencies were observed for the square and circular coils. Table III gives the comparison between the values of the measured resonant frequencies with losses (experimental measures) and those calculated theoretically (case without losses) for both coils.

TABLE III. THEORITICAL AND EXPERIMENTAL FREQUENCY COMPARISON

\begin{tabular}{|c|c|c|c|c|}
\hline \multirow{2}{*}{ Parameter } & \multicolumn{2}{|c|}{ Square Helmholtz coil } & \multicolumn{2}{c|}{ Circular Helmholtz coil } \\
\cline { 2 - 5 } & Theoretical & Experimental & Theoretical & Experimental \\
\hline $\begin{array}{c}\text { Resonant } \\
\text { frequency }\end{array}$ & $f_{0}=102 \mathrm{MHz}$ & $f_{0}=102 \mathrm{MHz}$ & $f_{0}=99 \mathrm{MHz}$ & $f_{0}=99 \mathrm{MHz}$ \\
\hline
\end{tabular}

It can be noted that the same value of the frequency $f_{0}$ is observed theoretically and experimentally. Impedance matching allows optimal energy transfer between the coils and the transmission and reception system. For an optimal transfer in the coaxial transmission cable during excitation, the impedance of the system should be $50 \Omega$ [14]. The matching of the coil is achieved by optimizing the position of the attack loop with the coils. Thus, the distance to have an impedance of $50 \Omega$ is equal to $2.77 \mathrm{~cm}$ and $2.56 \mathrm{~cm}$ for square and circular coils theoretically and practically.

\section{Test Results}

Comparative tests were conducted for both circular and square Helmholtz coils. In the air, in the absence of a sample, relative field measurements along the axis of the coils were conducted using a small test loop connected to the Network Analyzer as shown in Figure 10. A similar scheme was employed for the square coil with loops, as shown in Figure 11.

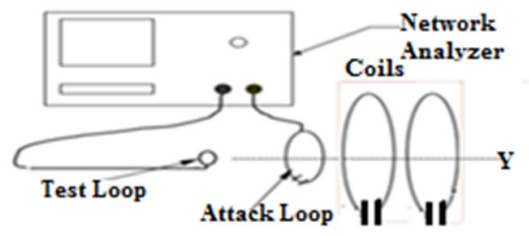

Fig. 10. The Mmasurement bench of the circular coil

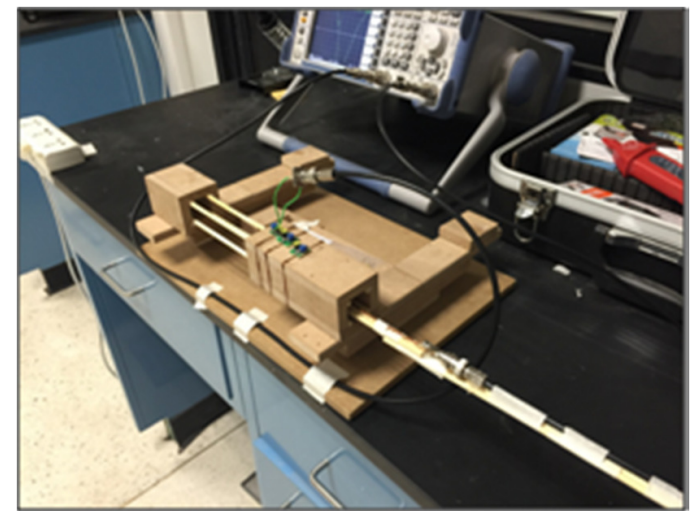

Fig. 11. Typical experimental setup for the square Helmholtz coil

The electromagnetic field along the axis of the coils (O-y) is measured with respect to the distance by facilitating to and fro motion arrangement as depicted in Figure 11. The curves of 
this normalized field produced by both coils are shown in Figure 12. This practical pattern can be compared with the simulation result depicted in Figure 5. Table IV illustrates the measurements of field intensity in terms of voltage with respect to the distance for a circular and a square Helmholtz coil. This practical result is plotted in Figure 12.

TABLE IV. MEASUREMENT OF FIELD INTENSITY IN TERMS OF VOLTAGE WITH RESPECT TO DISTANCE

\begin{tabular}{|c|c|c|c|c|}
\hline \multirow{2}{*}{$\begin{array}{c}\text { Distance } \\
(\mathbf{m} \mathbf{m})\end{array}$} & \multicolumn{2}{|c|}{ Circular coil } & \multicolumn{2}{c|}{ Square coil } \\
\cline { 2 - 5 } & Voltage (dB) & Voltage (V) & Voltage (dB) & Voltage (V) \\
\hline 24 & -5.04 & 0.313 & -4.86 & 0.327 \\
\hline 22 & -4.86 & 0.327 & -4.82 & 0.329 \\
\hline 20 & -4.62 & 0.345 & -4.78 & 0.332 \\
\hline 18 & -4.38 & 0.365 & -4.72 & 0.337 \\
\hline 16 & -4.16 & 0.384 & -4.66 & 0.342 \\
\hline 14 & -4.01 & 0.397 & -4.62 & 0.345 \\
\hline 12 & -3.76 & 0.421 & -4.57 & 0.349 \\
\hline 10 & -3.55 & 0.442 & -4.48 & 0.356 \\
\hline 8 & -3.38 & 0.459 & -4.42 & 0.361 \\
\hline 6 & -3.26 & 0.472 & -4.37 & 0.366 \\
\hline 4 & -3.23 & 0.474 & -4.32 & 0.369 \\
\hline 2 & -3.22 & 0.477 & -4.32 & 0.369 \\
\hline 0 & -3.22 & 0.477 & -4.32 & 0.369 \\
\hline-2 & -3.22 & 0.477 & -4.33 & 0.369 \\
\hline-4 & -3.24 & 0.474 & -4.35 & 0.367 \\
\hline-6 & -3.28 & 0.471 & -4.39 & 0.364 \\
\hline-8 & -3.34 & 0.463 & -4.46 & 0.358 \\
\hline-10 & -3.58 & 0.439 & -4.55 & 0.351 \\
\hline-12 & -3.78 & 0.419 & -4.62 & 0.345 \\
\hline-14 & -3.98 & 0.399 & -4.68 & 0.341 \\
\hline-16 & -4.22 & 0.378 & -4.74 & 0.336 \\
\hline-18 & -4.45 & 0.359 & -4.77 & 0.333 \\
\hline-20 & -4.68 & 0.341 & -4.83 & 0.329 \\
\hline-22 & -4.82 & 0.329 & -4.89 & 0.324 \\
\hline-24 & -5.11 & 0.308 & -4.91 & 0.323 \\
\hline & & & & \\
\hline
\end{tabular}

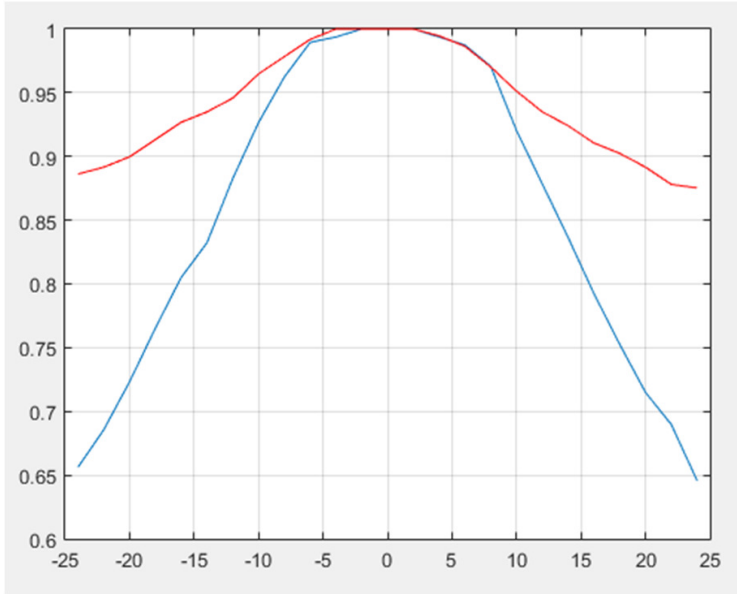

Fig. 12. Measurement results for the circular and square coils

\section{Results with Loaded Test for Circular and Square Coils}

The loaded tests for the developed coils were performed using Bruker-Biospec-Avance on MRI images which are in the form of a large open on the sides cylinder (dimensions: $1.40 \mathrm{~m}$ diameter and $1.2 \mathrm{~m}$ length). A cylindrical free region $(15 \mathrm{~cm}$ diameter) is used to receive the coil by the two lateral accesses. The static magnetic field produced had an intensity of $2.4 \mathrm{~T}$ and was directed along the horizontal axis, corresponding to the irradiation of the sample (water) at the Larmor frequency (v0 (proton) $=100.240 \mathrm{MHZ}$ ). An electrostatic or magnetic shielding was employed to prevent the coupling gradient coils and to improve the system's efficiency [19-20]. In the beginning, the coil was placed horizontally in the transverse plane of the spectrometer. The tuning of the coil with load was checked and refined. In the second step, after the verification of obtaining an NMR signal, a calibration of the homogeneity of the field was done by means of the shim coils provided for the purpose. In the last step, sections were made in imaging. Thus Figures 13-14 represent the axial sectional images obtained by the developed circular and square coils respectively.

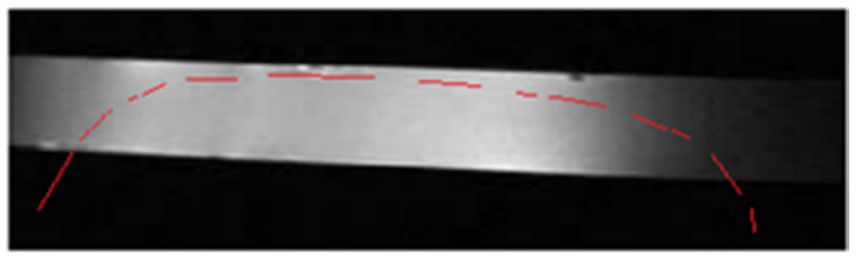

Fig. 13. Axial sectional image $(1 \mathrm{~mm})$ produced by the circular coil

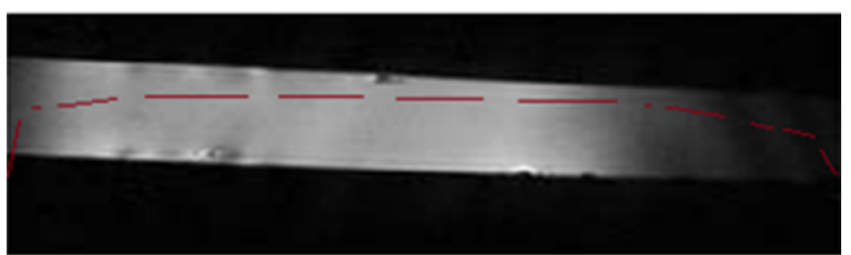

Fig. 14. Axial sectional image $(1 \mathrm{~mm})$ produced by the square coil

These images were utilized ( $1 \mathrm{~mm}$ slices) to extract relative profiles of the intensity of the images (Figure 13). Furthermore, the widths of the lobes noted for the developed coils are in proportions substantially identical to those found in the air without sample.

\section{CONCLUSION}

In this work, the operation of standard square and circular Helmholtz coils, which can be used for Magnetic Resonance Imaging, is investigated and demonstrated. The coil assembly comprises of two coaxial loops separated by a distance equal to their radius for the circular coil and half the length of the side for the square coil, and carrying the same current (co-current mode), in a symmetric arrangement. The developed Helmholtztype coils produce a second-order homogeneous field. Comparative study of simulation using MATLAB \& PSPICE along with the experimental implementation was conducted to check the magnetic field uniformity of the circular and square Helmholtz coils. The magnetic field value for which the coil was designed was attained successfully. Helmholtz coil is an excellent source of uniform magnetic field, and it was built within a very economic budget range. It was observed that if the radius of the circular coil is equal to the side of the square, and the distance between the loops is less than $50 \%$ of the radius for the circular loop and less than $55.4 \%$ of the side for the square loop, then the sensitivity of the magnetic field for the circular coil is more than for the square coil. But the pair of 
square coils exhibit greater homogeneity of magnetic field than the pair of circular coils. It was also observed that the distance between the loops must be approximately equal to $50 \%$ of the radius for the circular coil and $27.7 \%$ of the length of the side for the square Helmholtz coil. The pair of square Helmholtz coils exhibited more filed homogeneity than the circular Helmholtz coils, which is desired for MRI applications. The sensitivity (magnitude) of the magnetic field in the pair of the circular Helmholtz coils is found to be more than the one of the square coils.

\section{ACKNOWLEDGEMENT}

The authors would like to thank Mohammad Al Aqal for his participation throughout this work

\section{REFERENCES}

[1] F. Romeo, D. I. Hoult, "Magnet field profiling: analysis and correcting coil design", Magnetic Resonance in Medicine, Vol. 1, No. 1, pp. 44-65, 1984

[2] C. E. Hayes, W. A. Edelstein, J. F. Schenck, O. M. Mueller, M. Eash, "An efficient, highly homogeneous radiofrequency coil for whole-body NMR imaging at 1.5 T", Journal of Magnetic Resonance, Vol. 63, pp. 622-628, 1985

[3] L. Guendouz, S. M. A. Ghaly, A. Hedjiedj, J. M. Escanye, D. Canet, "Improved Helmholtz-type magnetic resonance imaging coils with highB1 homogeneity-spherical and ellipsoidal four-coil systems", Concepts in Magnetic Resonance, Vol. 33B, No. 1, pp. 9-20, 2008

[4] K. Asher, N. K. Bangerter, R. D. Watkins, G. E. Gold, "Radiofrequency coils for musculoskeletal MRI", Topics in Magnetic Resonance Imaging, Vol. 21, No. 5, pp. 315-323, 2010

[5] S. M. A. Ghaly, L. Guendouz, A. Hedjiedj, J. M. Escanye, D. Canet, "Improved Helmholtz-type coils with high B1 homogeneity-spherical and ellipsoidal configurations", 24th IASTED International MultiConference on Biomedical Engineering, Innsbruck, Austria, February 15-17, 2006

[6] S. Li, Q. X. Yang, M. B. Smith, "RF coil optimization: Evaluation of B1 field homogeneity using field histograms and finite element calculations", Magnetic Resonance Imaging, Vol. 12, No. 7, pp. 10791087, 1994

[7] B. Gruber, M. Froeling, T. Leiner, D. W. J. Klomp, "RF coils: A practical guide for nonphysicists", Journal of Magnetic Resonance, Vol. 48, No. 3, pp. 590-604, 2018

[8] J. Mispelter, M. Lupu, A. Briguet, NMR probeheads for biophysical and biomedical experiments, theoretical principles \& practical guidelines, 1 st edition, Imperial College Press, 2006

[9] B. J. Dardzinski, S. Li, C. M. Collins, G. D. Williams, M. B. Smith, "A birdcage coil tuned by RF shielding for application at $9.4 \mathrm{~T}$ ", Journal of Magnetic Resonance, Vol. 131, No. 1, pp. 32-38, 1998

[10] S. M. A. Ghaly, S. A. Sowayan, "A high B1 field homogeneity generation using free element elliptical four-coil system", American Journal of Applied Sciences, Vol. 11, No. 4, pp. 534-540, 2014

[11] S. M. A. Ghaly, K. A. A. Snaie, S. S. A. Sowayan, "Design and testing of radiofrequency spherical four coils", Modern Applied Science, Vol. 10, No. 5, pp. 186-193, 2016

[12] S. M. A. Ghaly, K. A. A. Snaie, O. K. Mohammad, "Spherical and improved Helmholtz coil with high B1 homogeneity for magnetic resonance imaging", American Journal of Applied Sciences, Vol. 13, No. 12, pp. 1413-1418, 2016

[13] S. M. A. Ghaly, K. A. A. Snaie, A. M. Ali, "Design and modeling of a radiofrequency coil derived from a Helmholtz structure", Engineering, Technology \& Applied Science Research, Vol. 9, No. 2, pp. 4037-4040, 2019

[14] M. Decorps, P. Blondet, H. Reutenauer, J. P. Albrand, C. Remy, "An inductively coupled, series-tuned NMR probe", Journal of Magnetic Resonance, Vol. 65, No. 1, pp. 100-109, 1985
[15] J. M. Boesch, A. P. Koretsky, "An in vivo NMR probe circuit for improved sensitivity”, Journal of Magnetic Resonance, Vol. 54, No. 3, pp. 526-532, 2003

[16] F. Alorifi, S. M. A. Ghaly, M. Shalaby, M. A. Ali, M. O. Khan, "Analysis and detection of a target gas system based on TDLAS \& labVIEW", Engineering, Technology \& Applied Science Research, Vol. 9, No. 3, pp. 4196-4199, 2019

[17] S. M. A. Ghaly, "LabVIEW based implementation of resistive temperature detector linearization techniques", Engineering, Technology \& Applied Science Research, Vol. 9, No. 4, pp. 4530-4533, 2019

[18] S. M. A. Ghaly, M. O. Khan, S. O. E. Mehdi, M. A. Awad, M. A. Ali, K. A. A. Snaie, "Implementation of a broad range smart temperature measurement system using auto-selected multi-sensor core in labVIEW environment", Engineering, Technology \& Applied Science Research, Vol. 9, No. 4, pp. 4511-4515, 2019

[19] D. M. Ginsberg, M. J. Melchner, "Optimum geometry of saddle shaped coils for generating a uniform magnetic field", Review of Scientific Instruments, Vol. 41, No. 1, pp. 122-123, 2010

[20] H. Fujita, T. Zheng, X. Yang, M. J. Finnerty, S. Handa, "RF surface receive array coils: The art of an LC circuit", Journal of Magnetic Resonance Imaging, Vol. 38, No. 1, pp. 12-25, 2013 